\title{
Generation of Gastroesophageal Reflux Disease Symptoms During Esophageal Acid Infusion With Concomitant Esophageal pH Monitoring in Healthy Adults
}

\author{
Shunji Ohara, ${ }^{1,2 *}$ Kenji Furuta, ${ }^{1}$ Kyoichi Adachi, ${ }^{3}$ Kousuke Fukazawa, ${ }^{1}$ Masahito Aimi, ${ }^{1}$ Masaharu Miki ${ }^{2}$ and Yoshikazu Kinoshita ${ }^{1}$ \\ Departments of ${ }^{1}$ Gastroenterology and Hepatology, and ${ }^{3}$ Clinical Nursing, Shimane University School of Medicine, Izumo, Japan; and \\ ${ }^{2}$ Division of Internal Medicine, Himeji Aiwa Hospital, Himeji, Japan
}

\section{Background/Aims}

The sensitivity of the upper and lower esophageal mucosa to acid is considered to differ. We investigated the relationship between $\mathrm{pH}$ changes in different sites of the esophagus and generation of gastroesophageal reflux symptoms during an acid infusion test.

\section{Methods}

An acid infusion catheter was placed at 5 or $15 \mathrm{~cm}$ above the lower esophageal sphincter (LES) in 18 healthy volunteers, while a 2-channel pH sensor catheter was also placed in each with the sensors set at 5 and $15 \mathrm{~cm}$ above the LES. Solutions containing water and hydrochloric acid at different concentrations were infused through the infusion catheter.

Results

Acid infusion in the upper esophagus caused a pH drop in both upper and lower esophageal sites, whereas that in the lower esophagus resulted in a significant pH drop only in the lower without a corresponding pH decline in the upper esophagus. Stronger heartburn, chest pain, and chest oppression symptoms were noted when acid was infused in the upper as compared to the lower esophagus, while increased intra-esophageal acidity strengthened each symptom. Regurgitations caused by upper and lower esophageal acid infusions were similar, and not worsened by a larger drop in intra-esophageal pH. Chest pain was caused only by lowered intra-esophageal pH, while heartburn, chest oppression, and regurgitation were induced by a less acidic solution.

\section{Conclusions}

Higher intra-esophageal acidity caused stronger heartburn, chest pain, and chest oppression symptoms. However, regurgitation was not significantly influenced by intra-esophageal acidity. The upper esophagus showed higher acid sensitivity than the lower esophagus.

(J Neurogastroenterol Motil 2013;19:503-508)

\section{Key Words}

Heartburn; Esophagus; Gastroesophageal reflux

Received: May 9, 2013 Revised: July 23, 2013 Accepted: July 31, 2013

(c) This is an Open Access article distributed under the terms of the Creative Commons Attribution Non-Commercial License (http://creativecommons. org/licenses/by-nc/3.0) which permits unrestricted non-commercial use, distribution, and reproduction in any medium, provided the original work is properly cited.

*Correspondence: Shunji Ohara, MD

Division of Internal Medicine, Himeji Aiwa Hospital, 3-219-1 lida, Himeji, Hyogo 670-0974, Japan

Tel: +81-79-234-2117, Fax: +81-79-234-2149, E-mail: shunjio777@yahoo.co.jp

Financial support: This work was supported by a research fund of Shimane University School of Medicine.

Conflicts of interest: None.

Author contributions: Shunji Ohara, Kenji Furuta, Kousuke Fukazawa and Masahito Aimi performed the research. Shunji Ohara, Kenji Furuta, Kyoichi Adachi, Kousuke Fukazawa, Masahito Aimi, Masaharu Miki and Yoshikazu Kinoshita designed the research study. Kenji Furuta, Kyoichi Adachi and Yoshikazu Kinoshita contributed essential tools. Shunji Ohara, Kenji Furuta, Kyoichi Adachi and Yoshikazu Kinoshita analyzed the data and wrote the manuscript. 


\section{Introduction}

Gastroesophageal acid reflux is the main triggering factor that causes gastroesophageal reflux disease (GERD) symptoms, such as heartburn and regurgitation, though the mechanisms that generate these symptoms are not fully understood. Based on previous studies that utilized 24-hour esophageal multiple $\mathrm{pH}$ monitoring and impedance-pH monitoring, the sensitivity of the upper and lower esophageal mucosa to gastroesophageal acid reflux is considered to differ, resulting in different symptom severity depending on the level reached by reflux contents. ${ }^{1-4}$ Acid infusion from an intra-luminal catheter at different sites of the esophagus also demonstrated that the sensitivity to acid is different in different parts of the esophagus. ${ }^{5,6}$ However, $\mathrm{pH}$ changes in different sites of the esophagus during acid infusion have not been investigated, nor has the co-relationship between intra-esophageal acidity and GERD symptoms during acid infusion been studied. We examined the relationship between upper and lower esophageal $\mathrm{pH}$ changes, and generation of GERD symptoms during intra-esophageal acid infusion.

\section{Materials and Methods}

Eighteen healthy volunteers (10 males, 8 females; mean age, $25.6 \pm 2.0$ years) without gastrointestinal symptoms were enrolled as study subjects. None had a history of gastrointestinal surgery or administration of drugs that might influence gastrointestinal motility or acid secretion. All subjects underwent an upper gastrointestinal endoscopy examination within 3 months prior to the study to ensure that there were no lesions.

The subjects were instructed and confirmed that heartburn was defined as a burning sensation in the retrosternal area, regurgitation was a sensation of upward movement of liquid into the mouth, hypopharynx, or upper esophagus, and chest oppression was a sensation that something is caught or jammed in the esophagus.

After at least 4 hours of fasting, the location of the lower esophageal sphincter (LES) was determined using manometry, then a thin nasogastric feeding tube was inserted in a transnasal manner into the esophagus and placed at 5 (lower esophagus) or 15 (upper esophagus) $\mathrm{cm}$ above the LES. A 2-channel $\mathrm{pH}$ sensor catheter was also inserted transnasally into the esophagus after calibration at $\mathrm{pH} 1.0$ and 7.0, according to the instructions of the manufacturer, and placed to set the sensors at 5 and $15 \mathrm{~cm}$ above the LES. The subjects sat in a stable chair with a $45^{\circ}$ angled back and rested for 30 minutes, then distilled water at room temperature was infused for 15 minutes at a constant speed of $5 \mathrm{~mL} / \mathrm{mi}$ nute with a syringe pump. Next, without informing the subject of the timing of the switch from distilled water, 7 different solutions with varying concentrations of hydrochloric acid $(\mathrm{HCl})\left(10^{-7}\right.$ to $10^{-1} \mathrm{M}$ ) at room temperature were serially infused at a speed of 5 $\mathrm{mL} /$ minute for 3 minutes at each concentration, starting with the lowest. The subjects recorded the severity of 4 GERD symptoms (heartburn, chest pain, chest oppression and regurgitation) each minute using a visual analog scale. A $10 \mathrm{~cm}$ rating line was used to rate 'no symptoms' at 0 to 'worst level of symptoms' at $10 \mathrm{~cm}$. The subjects were not informed regarding the intra-esophageal sites of acid infusion, types of infused solutions, or timing of the alteration of solutions. In addition, subjects were instructed to spit saliva without swallowing during the test. When they had difficulty with spitting saliva, a piece of gauze was kept in the mouth to prevent the swallowing. These esophageal acid infusion tests in the upper and lower esophagus were performed in all subjects on separate days using a crossover design with an interval of at least 1 week.

The study was performed according to the World Medical Association Declaration of Helsinki. The study protocol was approved by the ethical committee of Shimane University School of Medicine and written informed consent was obtained from each of the enrolled subjects.

\section{Statistical Methods}

Data are expressed as the mean $\pm \mathrm{SE}$. Statistical analysis was performed using a paired $t$ test for comparisons of paired data. All calculations were done with the Stat View 5.0 software package for Macintosh (Abacus Concepts Inc, Berkeley, CA, USA). Differences at $P<0.05$ were considered to be statistically significant.

\section{Results}

We successfully measured $\mathrm{pH}$ changes in the esophagus during acid infusion in different sites of the esophagus in all 18 study subjects without any adverse events. Acid infusion in the upper esophagus caused dose-dependent $\mathrm{pH}$ drops in both the upper and lower esophagus (Fig. 1A). In contrast, infusion in the lower esophagus resulted in a significant $\mathrm{pH}$ drop only in the lower esophagus without a corresponding $\mathrm{pH}$ decline in the upper esophagus (Fig. 1B). 
A

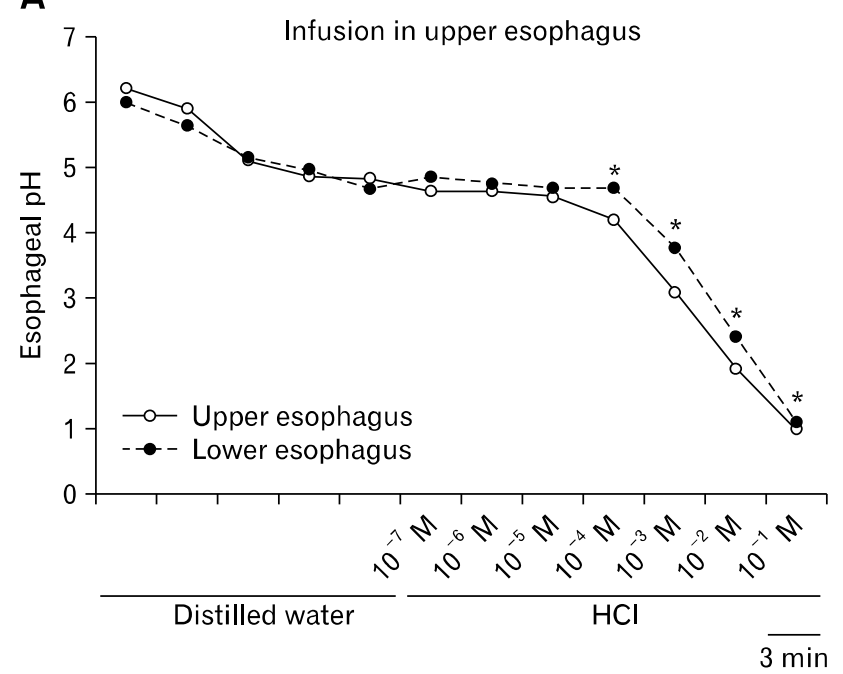

B

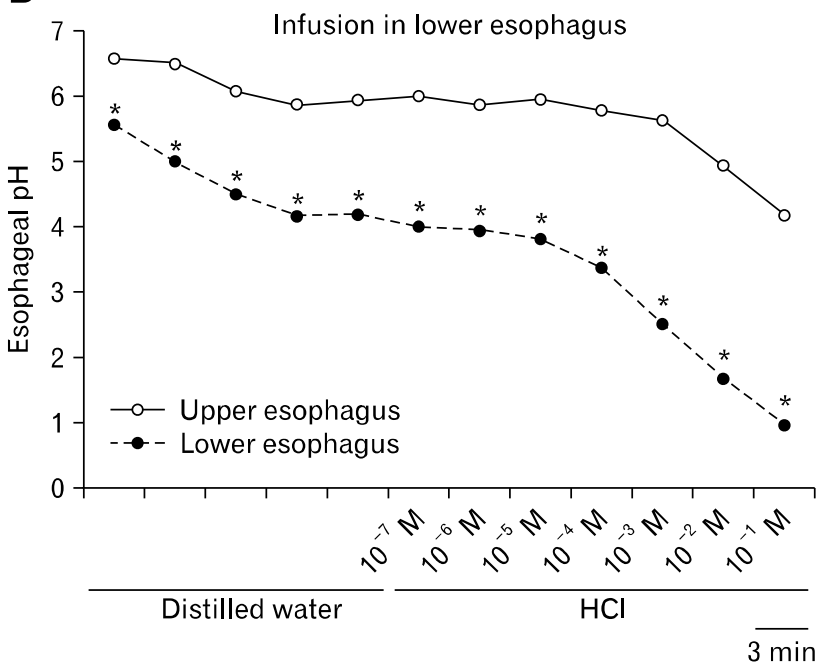

Figure 1. pH changes in upper (black open circles) and lower (black closed circles) esophagus during water and acid infusion in the (A) upper and (B) lower esophagus. The vertical axis shows the mean $\mathrm{pH}$ values during a 3-minute infusion of the same solution, while the horizontal axis shows the concentration of the infused hydrochloric acid $(\mathrm{HCl})$ solution. The concentration of the $\mathrm{HCl}$ solution is higher toward the right, with $0.1 \mathrm{M} \mathrm{HCl}$ the highest value. Each dot represents a mean value. ${ }^{*} P<0.05$, significant difference between upper and lower esophagus.

The severity of heartburn during acid infusion in the upper esophagus was significantly higher than that in the lower esophagus (Fig. 2A). Furthermore, the severity of chest pain and chest oppression symptoms during acid infusion in the upper esophagus was also higher than that in the lower esophagus, though the difference did not reach a statistically significant level (Fig. 2B and $2 \mathrm{C}$ ). These 3 symptoms became stronger with infusion of higher acidic solutions. During acid infusion in the lower esophagus, the severity of heartburn and chest pain was remarkably increased only after the infusion of $10^{-1} \mathrm{M} \mathrm{HCl}$, the highest acidic level, suggesting a higher sensory threshold to acid in the lower esophagus. In contrast, regurgitations caused by upper and lower esophageal acid infusion were similar, and their severity did not remarkably change with infusion of a higher acidic solution (Fig. 2D). These results suggest that regurgitation is induced regardless of the $\mathrm{pH}$ value, different from heartburn and chest pain symptoms.

To compare the level of acidity that causes each symptom, the mean intra-esophageal $\mathrm{pH}$ levels that initially caused the symptoms were calculated, with the results shown in Figure 3. During acid infusion in the lower esophagus, chest pain was initiated at a lower intra-esophageal $\mathrm{pH}$ level as compared to heartburn $(P=$ $0.005)$, chest oppression $(P=0.028)$ and regurgitation $(P=$ $0.035)$.

\section{Discussion}

There are several investigations for factors that influence generation of GERD symptoms, including duration of acid reflux events, lower nadir $\mathrm{pH}$, large $\mathrm{pH}$ drop and proximal extent of acidic refluxate. ${ }^{1-4}$ The sensitivity to acid has been suggested to differ between the upper and lower esophageal mucosa in studies that utilized acid infusion in the esophagus. ${ }^{5,6}$ To the best of our knowledge, this is the first study to demonstrate a relationship between $\mathrm{pH}$ changes in different sites of the esophagus and generation of GERD symptoms during acid infusion in the upper and lower esophagus. The status of Helicobacter pylori infection was not examined in this study, since it is not considered to be an important factor for determining baseline intra-esophageal $\mathrm{pH}$ in normal asymptomatic volunteers.

Smith et $\mathrm{al}^{7}$ investigated symptoms generated by infusions of different concentrations of $\mathrm{HCl}$ solutions in the lower esophagus and reported that the incidence of pain increased with increasing acidity in the lower esophagus. Our results showed that lower intra-esophageal $\mathrm{pH}$ was related to more severe heartburn, chest pain and chest oppression, not only during acid infusion in the upper esophagus but also that in the lower esophagus. Therefore, those 3 symptoms may be generated by hydrogen-ion sensitive receptors. In contrast, the severity of regurgitation was not augmented by increased acidity of the infused solution. It was re- 
A

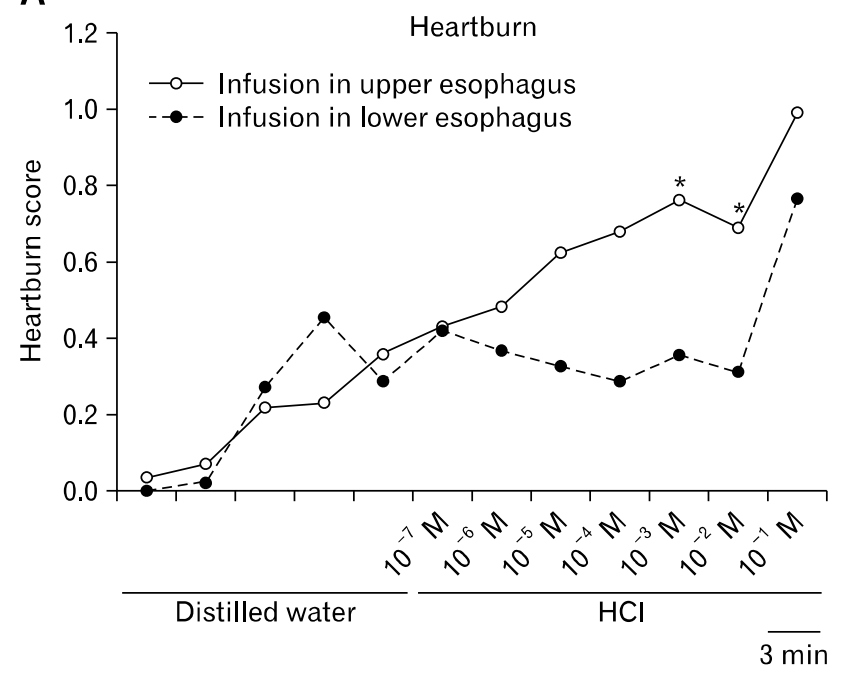

C

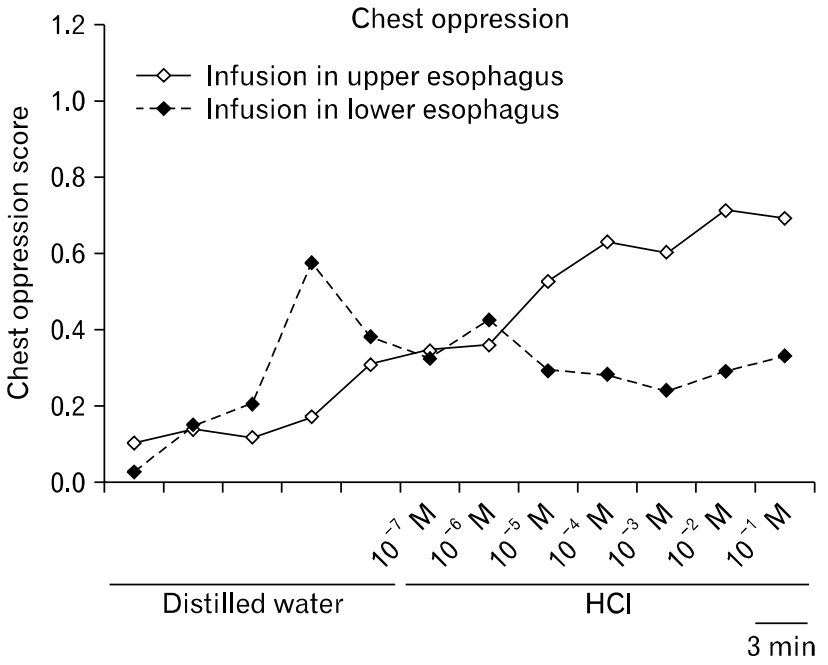

B

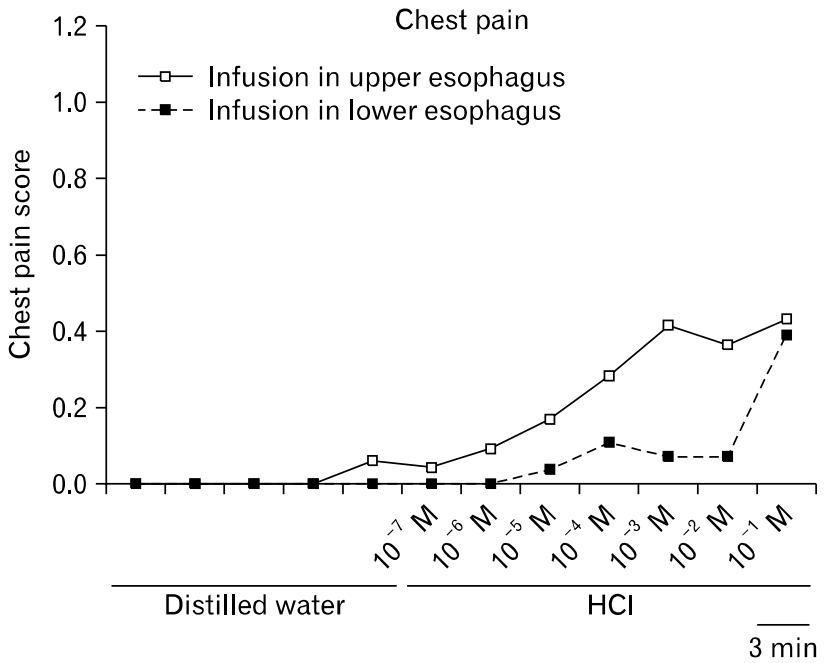

D

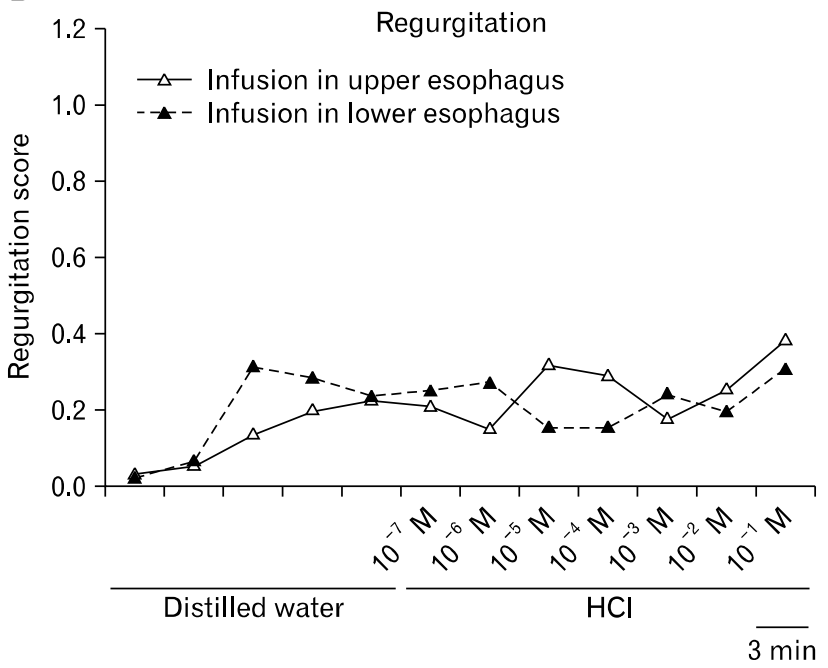

Figure 2. Generation of reflux symptoms during water and acid infusion in the upper (black open markers) and lower (black closed markers) esophagus. The vertical axis shows the mean scores for reflux symptoms (A-D) during a 3-minute infusion of the same solution, while the horizontal axis shows the concentration of the infused hydrochloric acid $(\mathrm{HCl})$ solution. The concentration of the $\mathrm{HCl}$ solution is higher toward the right, with $0.1 \mathrm{M} \mathrm{HCl}$ the highest value. ${ }^{*} P<0.05$, significantly different between upper and lower infusion.

cently reported that regurgitation is less responsive to acid suppression therapy than heartburn in GERD patients. ${ }^{8}$ Therefore, the mechanism related to sense of regurgitation may be different from that of heartburn. Regurgitation may be more highly associated with the volume of refluxate in the esophagus than acidity, though we were unable to investigate the influence of speed or volume of the infused solution on generation of regurgitation in the present study. In a separate study, we investigated possible pooling of the infused solution in the esophagus in 2 subjects. When a diluted contrast agent was infused into the esophagus at the same speed and position as in the present study, fluoroscopic imaging showed that most of the infused solution flowed into the stomach without remaining in the esophagus. Therefore, we consider that the infused fluid did not remain in the esophagus in the present subjects and generation of GERD symptoms was not affected by that infusion.

The severity of heartburn, chest pain, and chest oppression during acid infusion in the upper esophagus was higher than that during acid infusion in the lower esophagus, though $\mathrm{pH}$ in the lower esophagus during infusion in the lower esophagus was similar to that during infusion in the upper esophagus. Therefore, the sensitivity to heartburn, chest pain, and chest oppression in 


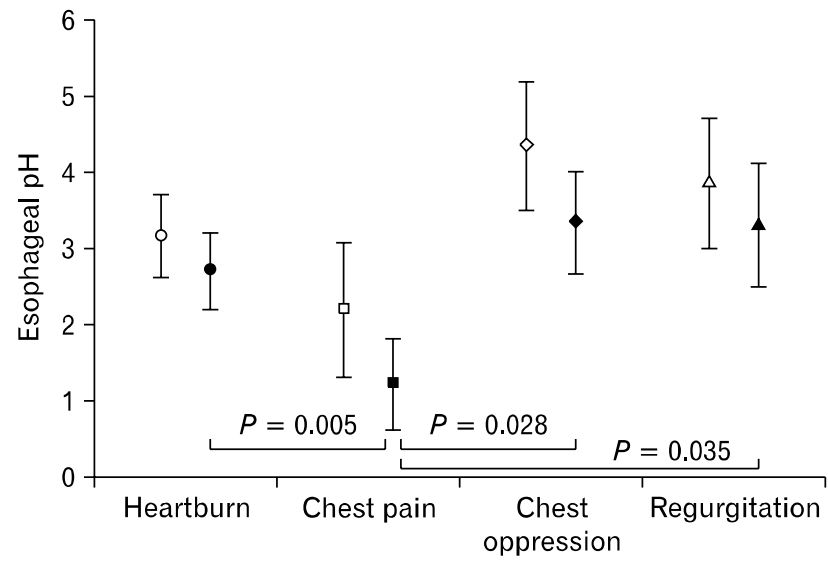

Figure 3. Mean intra-esophageal $\mathrm{pH}$ value that causes symptoms. Open markers represent the mean upper esophageal $\mathrm{pH}$ to initially cause symptoms during upper esophageal infusion and closed markers represent the mean lower esophageal $\mathrm{pH}$ to initially cause symptoms during lower esophageal infusion. Each symptom showed a different threshold to the acidic solution. Higher acidity tended to be necessary to cause symptoms when the solution was infused in the lower esophagus. Vertical lines represent the mean $\pm \mathrm{SE}$.

the esophageal mucosa of the upper esophagus is considered to be greater than that in lower esophagus, as previous studies have also suggested. ${ }^{1-6}$ In rats, a denser supply of vagal mucosal afferent fibers was found in the upper esophagus, while sensory signals detected by specialized sensory structures were reported to travel mainly through the superior laryngeal nerve to their perikarya located in the cranial part of the vagal sensory ganglionic complex. Thus, the differences in sensitivity to heartburn, chest pain, and chest oppression may be caused by variations in mucosal innervations between the upper and lower esophagus, though no information from human studies is presently available.

This study has several limitations. First, the subjects were young healthy volunteers without GERD symptoms, and sensitivity to acid has been reported to be different among patients with non-erosive reflux disease, patients with reflux esophagitis, and normal individuals. ${ }^{10,11}$ The present symptom scores observed during acid infusion were consistent among the subjects and varied only slightly, as they were asymptomatic healthy volunteers. Therefore, further investigation using GERD patients is needed to clarify the relationship between $\mathrm{pH}$ changes in different sites of the esophagus and generation of GERD symptoms during acid infusion, though a difference in sensitivity to acid between the upper and lower esophagus is also considered to be present in those patients. ${ }^{1-5}$ It was previously reported that an esophageal acid infusion induced stronger reflux symptoms in
GERD patients compared to healthy controls. ${ }^{12}$ Indeed, the symptoms observed in our study were not strong. However, because of the normal architecture of the esophageal mucosa present in our subjects, the symptomatic responses were considered to be more consistent. This may be the reason for the consistent results of the present study, even though the symptoms caused by acid infusion were not strong. Some of the present subjects did not report any GERD symptoms during acid infusion. Thus, quicker infusions and higher volumes may be necessary in the future study for resistant subjects. Second, acid infusion in the upper esophagus also provokes mucosal exposure to acid in the lower esophagus. A larger area of esophageal mucosa exposed to acid may cause stronger GERD symptoms, since a larger number of acid sensors present on the esophageal mucosa are considered to be stimulated. Infusion into the lower esophagus is the standard procedure used for esophageal acid infusion testing, ${ }^{9,13}$ thus acid infusion in the upper esophagus was considered to simultaneously stimulate the upper and lower esophagus, while that in the lower esophagus stimulated only the lower esophagus in the present subjects. Further study may be necessary with a novel method that selectively and separately stimulates the upper and the lower esophagus.

In conclusion, we found that sensitivity to acid in the upper esophagus was higher than that in the lower esophagus in our normal subjects. Furthermore, in contrast to other reflux symptoms, regurgitation was not augmented by increasing the acidity of the infused solution.

\section{References}

1. Weusten BL, Akkermans LM, vanBerge-Henegouwen GP, Smout AJ. Symptom perception in gastroesophageal reflux disease is dependent on spatiotemporal reflux characteristics. Gastroenterology 1995;108:1739-1744.

2. Cicala M, Emerenziani S, Caviglia R, et al. Intra-oesophageal distribution and perception of acid reflux in patients with non-erosive gastro-oesophageal reflux disease. Aliment Pharmacol Ther 2003; 18:605-613.

3. Bredenoord AJ, Weusten BL, Curvers WL, Timmer R, Smout AJ. Determinants of perception of heartburn and regurgitation. Gut 2006;55:313-318.

4. Emerenziani S, Ribolsi M, Sifrim D, Blondeau K, Cicala M. Regional oesophageal sensitivity to acid and weakly acidic reflux in patients with non-erosive reflux disease. Neurogastroenterol Motil 2009;21:253-258.

5. Thoua NM, Khoo D, Kalantzis C, Emmanuel AV. Acid-related oesophageal sensitivity, not dysmotility, differentiates subgroups of patients with non-erosive reflux disease. Aliment Pharmacol Ther 2008;27:396-403. 
6. Dutta SK, Agrawal K, Mahmoud MA. Modulation of salivation and heartburn in response to the site of acid infusion in the human oesophagus. Aliment Pharmacol Ther 2010;32:795-800.

7. Smith JL, Opekun AR, Larkai E, Graham DY. Sensitivity of the esophageal mucosa to $\mathrm{pH}$ in gastroesophageal reflux disease. Gastroenterology 1989;96:683-689.

8. Kahrilas PJ, Jonsson A, Denison H, Wernersson B, Hughes N, Howden CW. Regurgitation is less responsive to acid suppression than heartburn in patients with gastroesophageal reflux disease. Clin Gastroenterol Hepatol 2012;10:612-619.

9. Wank M, Neuhuber WL. Local differences in vagal afferent innervation of the rat esophagus are reflected by neurochemical differences at the level of the sensory ganglia and by different brainstem projections. J Comp Neurol 2001;435:41-59.
10. Miwa $\mathrm{H}$, Minoo T, Hojo $\mathrm{M}$, et al. Oesophageal hypersensitivity in Japanese patients with non-erosive gastro-oesophageal reflux diseases. Aliment Pharmacol Ther 2004;20(suppl 1):112-117.

11. Nagahara A, Miwa H, Minoo T, et al. Increased esophageal sensitivity to acid and saline in patients with nonerosive gastro-esophageal reflux disease. J Clin Gastroenterol 2006;40:891-895.

12. Szczesniak MM, Fuentealba SE, Cook IJ. Acid sensitization of esophageal mucosal afferents: implication for symptom perception in patients across the gastroesophageal reflux disease spectrum. Clin J Pain 2013;29:70-77.

13. Grade A, Pulliam G, Johnson C, Garewal H, Sampliner RE, Fass R. Reduced chemoreceptor sensitivity in patients with Barrett's esophagus may be related to age and not to the presence of Barrett's epithelium. Am J Gastroenterol 1997;92:2040-2043. 